\title{
A PROBLEM IN LINEAR DIFFERENTIAL EQUATIONS ${ }^{1}$
}

TOMLINSON FORT

In the Bulletin of the American Mathematical Society, March 1958, page 61, R. E. Bellman suggests the following for research.

"Consider the second order linear differential equation

$$
u^{\prime \prime}+(1+\lambda g(x)) u=0
$$

where $\lambda$ is a real constant and $\int_{0}^{\infty}|g(x)| d x<\infty$. Let $u_{1}(x)$ be the solution specified by $u_{1}(0)=0, u_{1}^{\prime}(0)=1$. It is known that

$$
u_{1}(x) \sim r(\lambda) \sin (x+\theta(\lambda)) .
$$

Taking $\lambda$ to be a complex variable, what are the analytic properties of the functions $r(\lambda)$ and $\theta(\lambda)$ ? In particular, where are the singularities nearest the origin? If $g(x)>0$ for $x \geqq 0$, is the singularity nearest the origin on the negative axis?" We assume that $g(x)$ is real for real $x$.

1. It is known ${ }^{2}$ that there are two solutions of (1) which we denote by $u_{3}(x)$ and $u_{4}(x)$ such that when $x \rightarrow \infty$

$$
\begin{aligned}
u_{3}(x)-\sin x & \rightarrow 0, \\
u_{3}^{\prime}(x)-\cos x & \rightarrow 0 . \\
u_{4}(x)-\cos x & \rightarrow 0, \\
u_{4}^{\prime}(x)+\sin x & \rightarrow 0 .
\end{aligned}
$$

This approach is uniform in $\lambda$ so long as $|\lambda|<M$. This last statement does not seem to have been made in the literature. However, it is readily inferred from text book proofs.

2. Let $u_{1}(x)$ and $u_{2}(x)$ be solutions of (1) such that

$$
\begin{array}{ll}
u_{1}(0)=0, & u_{1}^{\prime}(0)=1, \\
u_{2}(0)=1, & u_{2}^{\prime}(0)=0 .
\end{array}
$$

Choose $b>0$. Let $U_{1}(x)$ and $U_{2}(x)$ be solutions of (1) such that

$$
\begin{array}{ll}
U_{1}(b)=\sin b, & U_{1}^{\prime}(b)=\cos b, \\
U_{2}(b)=\cos b, & U^{\prime}(b)=-\sin b .
\end{array}
$$

The two functions $u_{1}(x)$ and $u_{2}(x)$ form a fundamental system of solu-

Presented to the Society, November 29, 1958; received by the editors August 22, 1958.

1 This research was supported by the United States Air Force.

2 Coddington and Levinson, Theory of ordinary differential equations, McGrawHill, 1955, p. 92. 
tions of (1). Similarly $u_{3}(x)$ and $u_{4}(x)$ form a fundamental system and likewise $U_{1}(x)$ and $U_{2}(x)$.

Since $u_{3}(x), u_{3}^{\prime}(x), u_{4}(x), u_{4}^{\prime}(x)$ are bounded, all solutions of (1) and their first and second derivatives are bounded as is already well known. We write

$$
\begin{aligned}
& u_{1}(x)=c_{11}^{(b)}(\lambda) U_{1}(x)+c_{12}^{(b)}(\lambda) U_{2}(x) \\
& u_{2}(x)=c_{21}^{(b)}(\lambda) U_{1}(x)+c_{22}^{(b)}(\lambda) U_{2}(x)
\end{aligned}
$$

and

$$
\begin{aligned}
& u_{1}(x)=e_{11}(\lambda) u_{3}(x)+e_{12}(\lambda) u_{4}(x), \\
& u_{2}(x)=e_{21}(\lambda) u_{3}(x)+e_{22}(\lambda) u_{4}(x) .
\end{aligned}
$$

We note that the $c$ 's are independent of $x$.

We shall prove the following theorem:

Theorem 1. The functions $c_{i j}(\lambda), i, j=1,2$ can be analytically continued for complex $\lambda$ so that each is an analytic function of $\lambda$ so long as $|\lambda| \leqq M$, where $M$ is arbitrary.

We form the difference equation in discrete argument

$$
\begin{gathered}
(n / b)^{2} \Delta^{2} y\left(x_{i}\right)+\left[1+\lambda g\left(x_{i}\right)\right] y\left(x_{i+1}\right)=0, \\
x_{0}=0, \quad x_{i+1}=x_{i}+b / n, \quad \Delta y\left(x_{i}\right)=y\left(x_{i+1}\right)-y\left(x_{i}\right) .
\end{gathered}
$$

Here $i$ and $n$ are integers. Let $y_{1}\left(x_{i}\right)$ and $y_{2}\left(x_{i}\right)$ be those solutions of (7) such that

$$
\begin{aligned}
y_{1}(0) & =0, & n / b \Delta y_{1}(0) & =1, \\
y_{2}(0) & =1, & \Delta y_{2}(0) & =0 .
\end{aligned}
$$

Also let $Y_{1}\left(x_{i}\right)$ and $Y_{2}\left(x_{i}\right)$ be solutions of (7) such that

$$
\begin{array}{ll}
Y_{1}(b)=\sin b, & \Delta Y_{1}(b)=\Delta \sin b, \\
Y_{2}(b)=\cos b, & \Delta Y_{2}(b)=\Delta \cos b .
\end{array}
$$

Now count backward from $b$. By the use of (7) we find that $Y_{1}(0)$ $\Delta Y_{1}(0), Y_{2}(0), \Delta Y_{2}(0)$ are all polynomials in $\lambda$. We now write

$$
\begin{gathered}
y_{1}\left(x_{i}\right)=k_{11}^{(b)}(\lambda) Y_{1}\left(x_{i}\right)+k_{12}^{(b)}(\lambda) Y_{2}\left(x_{i}\right), \\
\Delta y_{1}\left(x_{i}\right)=k_{11}^{(b)}(\lambda) \Delta Y_{1}\left(x_{i}\right)+k_{12}^{(b)}(\lambda) \Delta Y_{2}\left(x_{i}\right)
\end{gathered}
$$

and

$$
\begin{aligned}
y_{2}\left(x_{i}\right) & =k_{21}^{(b)}(\lambda) Y_{1}\left(x_{i}\right)+k_{22}^{(b)}(\lambda) Y_{2}\left(x_{i}\right), \\
\Delta y_{2}\left(x_{i}\right) & =k_{21}^{(b)}(\lambda) \Delta Y_{1}\left(x_{i}\right)+k_{22}^{(b)}(\lambda) \Delta Y_{2}\left(x_{i}\right) .
\end{aligned}
$$


The $k$ 's are independent of $x_{i}$. Choose $x_{i}=0$. Note that $Y_{1}\left(x_{i}\right) \Delta Y_{2}\left(x_{i}\right)$ $-Y_{2}\left(x_{i}\right) \Delta Y_{1}\left(x_{i}\right)$ is a constant and can be evaluated at $b$. Solve for the $k$ 's. We find

$$
k_{11}^{(b)}(\lambda)=(b / n \csc b / n) Y_{2}(0)
$$

with similar forms for the other $k$ 's. These forms show that the $k$ 's are polynomials in $\lambda$. If we solve (5) for the $c$ 's with $x=0$ we find

$$
c_{11}^{(b)}(\lambda)=U_{2}(0) \text {. }
$$

We make all solutions of (7) functions of the continuous variable $x$ by connecting $y\left(x_{i}\right)$ and $y\left(x_{i+1}\right)$ when plotted in the Cartesian plane by straight line segments. We write $y(x)$.

Now let $n \rightarrow \infty$ holding $b$ fixed. As is well known the $y$ 's approach the corresponding $u$ 's uniformly in $x$ and $\lambda$ when $0 \leqq x \leqq b$ and $|\lambda|$ $\leqq M$. Hence $Y_{2}(0) \rightarrow U_{2}(0)$ uniformly in $\lambda$. Hence $k_{i j}^{(b)}(\lambda) \rightarrow c_{i j}^{(b)}(\lambda)$, $i, j=1,2$ uniformly in $\lambda$. Hence the $c_{i j}^{(b)}(\lambda)$ are analytic in $\lambda$ when $|\lambda| \leqq M$.

3. We now let $b \rightarrow \infty$. As already noted the $c$ 's are independent of $x$ and can be determined at any point. With the aid of (4) and (5) we find

$$
c_{11}^{(b)}(\lambda)=u_{1}(b) \sin b+u_{1}^{\prime}(b) \cos b .
$$

Let $u_{3}(x)=\sin x+\epsilon(x), u_{4}(x)=\cos x+\eta(x)$ then from (6) $c_{11}(\lambda)=\frac{u_{1}(b)\left[\sin b-\eta^{\prime}(b)\right]+u_{1}^{\prime}(b)[\cos b+\eta(b)]}{[\sin b+\epsilon(b)]\left[\sin b-\eta^{\prime}(b)\right]+[\cos b+\eta(b)]\left[\cos b+\epsilon^{\prime}(b)\right]}$.

Now $\epsilon(b), \eta(b), \epsilon^{\prime}(b), \eta^{\prime}(b)$ approach zero uniformly in $\lambda$ and $u(b)$ and $u^{\prime}(b)$ are bounded. Hence $\left(c_{11}(\lambda) \rightarrow c_{11}^{(b)}(\lambda)\right) \rightarrow 0$ uniformly in $\lambda$. Since $c_{11}^{(b)}(\lambda)$ is analytic, $|\lambda|<M$, so is $c_{11}(\lambda)$. The same reasoning applies to $c_{12}(\lambda), c_{21}(\lambda)$ and $c_{22}(\lambda)$.

This completes the proof of Theorem 1.

4. We know

$$
\begin{aligned}
u_{1}(x) & =c_{11}(\lambda) u_{3}(x)+c_{12}(\lambda) u_{4}(x) \sim c_{11}(\lambda) \sin x+c_{12}(\lambda) \cos x \\
& =r(\lambda) \sin (x+\theta(\lambda)), \quad|\lambda|<M
\end{aligned}
$$

where

$$
\begin{aligned}
{[r(\lambda)]^{2} } & =\left[c_{11}(\lambda)\right]^{2}+\left[c_{12}(\lambda)\right]^{2}, \\
\cos \theta(\lambda) & =c_{11}(\lambda) / r(\lambda) .
\end{aligned}
$$

so long as $\lambda$ is real. If we analytically continue the functions $[r(\lambda)]^{2}$ 
and $\cos \theta(\lambda)$ allowing $\lambda$ to be complex we observe that $[r(\lambda)]^{2}$ is entire and that the only possible singularities of $\cos \theta(\lambda)$ are where $r(\lambda)=0$. Now the Wronskian of $u_{3}(x)$ and $u_{4}(x)$ is a constant. It approaches -1 and consequently is -1 . With this in mind with the use of (3) and (6) we find $c_{11}(\lambda)=u_{4}(0), c_{12}(\lambda)=-u_{3}(0)$. Hence $c_{11}(\lambda)$ and $c_{12}(\lambda)$ are not both zero else $u_{3}(x)$ and $u_{4}(x)$ would have a Wronskian equal to zero which they do not. We conclude among other things that if $r(\lambda)=0$ then $c_{11}(\lambda) \neq 0, c_{12}(\lambda) \neq 0$.

Let $\lambda$ be real. Then $c_{11}(\lambda)$ and $c_{12}(\lambda)$ are real and $r(\lambda)$ can not vanish unless both vanish simultaneously. Hence the zeros of $r(\lambda)$ are all imaginary. We note in passing that $r(0)=1$.

We have proved the following theorem:

THEOREM 2. If $[r(\lambda)]^{2}$ and $\cos \theta(\lambda)$ are analytically continued in the complex plane, then $[r(\lambda)]^{2}$ is an entire function and $\cos \theta(\lambda)$ is in general analytic. Its only finite singularities are at the points where $r(\lambda)=0$. Such points are necessarily imaginary.

University of South Carolina 